\title{
Detection of Amp-C Beta Lactamase Enzyme Production among Enterobacteriaceae and Comparison of Different Inducer Substrate Combinations for Detection of Inducible Amp-C
}

\author{
Sindhu Cugati ${ }^{1}$ and P.R. Lyra ${ }^{2}$ \\ ${ }^{1}$ Department of Microbiology, Sree Balaji Medical College and Hospital, Chennai, India \\ ${ }^{2}$ Department of Microbiology, East Point College of Medical Sciences, Bangalore, India \\ *Corresponding author
}

\begin{tabular}{|l|}
\hline Ke y w o r d s \\
B-lactamases, \\
AmpC, \\
Cephalosporinases, \\
Boronic acid, \\
Inducers \\
\hline Article Info \\
\hline $\begin{array}{l}\text { Accepted: } \\
\text { 07 March } 2019 \\
\text { Available Online: } \\
\text { 10 April } 2019\end{array}$ \\
\hline
\end{tabular}

\section{A B S T R A C T}

AmpC beta-lactamases hydrolyse penicillins, monobactams, cephalosporins and cephamycins. AmpC producers are resistant tobetalactam/betalactamase inhibitor combinations therapeutically. AmpC is generally underreported which leads to therapeutic failures and uncontrolled spread of these resistant strains. Hence, there is an increased need to detect AmpC routinely in the laboratory. To detect AmpC $\beta$-lactamase production among Enterobacteriaceae isolated from clinical samples and to compare different inducer substrate combinations for the detection of inducible Amp-C (iAmpC). 100 clinical isolates of Enterobacteriaceae were tested. Constitutive AmpC (cAmpC) detected using inhibitor based method using Cefoxitin $(\mathrm{CN})$ and $\mathrm{CN}$ with Phenylboronic acid (PBA). Inducible $\mathrm{AmpC}$ detected using disk approximation test using inducers Imipenen (I) and Cefoxitin (CN), and substrates Cefotaxime (CTX), Ceftazidime (CAZ) and PiperacillinTazobactum (PT). Various combinations tested were I/PT, I/CTX, I/CAZ, CN/CTX, $\mathrm{CN} / \mathrm{CAZ}$. AmpC production was detected in $30 \%$ of isolates, $23 \%$ were constitutive and 7\% were inducible. Commonest AmpC producer was Enterobacter sp with 7(36.84\%) and 4(21.05\%), followed by E. coli $14(26.92 \%)$ and 3(5.76\%) constitutive and iAmpC respectively. 2(8.69\%) Klebsiella demonstrated only cAmpC. I/PT combination detected all the $7 \mathrm{iAmpC}$, others I/CTX and I/CAZ detected only 3isolates. Simple disk method of cefoxitin with boronic acid and I/PT combination can be used to detect constitutive and inducible AmpC respectively.

\section{Introduction}

$\beta$-lactamases are clinically important cephalosporinases encoded on the chromosomes of many Enterobacteriaceae and a few other organisms, where they mediate resistance to cephalothin, cefazolin, cefoxitin, most penicillins, and $\beta$-lactamase inhibitor/ $\beta$-lactam combinations(Gunjan Gupta et al., 2014). Isolates that coproduce both an Extended spectrum $\beta$-lactamase (ESBL) and a high level of AmpC are becoming more common (Naveen Grover., 2013). Mechanism of drug resistance in 
AmpC $\beta$ lactamase can be chromosomal or plasmid mediated. Chromosomal mediated resistance is due to mutation in the nucleotide sequence at some point of the DNA of the bacteria and such genes are not easily transferable to other bacterial species. Plasmid mediated AmpC $\beta$ lactamases have arisen by the transfer of chromosomal genes for AmpC $\beta$-lactamase onto plasmids. These genetic determinants can spread laterally and to other bacteria through lateral transfer of plasmids (Ascelijn Reuland, 2015).

Majority of AmpC $\beta$ lactamases are chromosomally mediated (Unlike ESBLs which are plasmid mediated) and are found in SPACE bugs (Serratia, Pseudomonas, Acinetobacter, Citrobacter and Enterobacter spp.). Plasmid mediated AmpC $\beta$ lactamases are seen in isolates of E.coli, $K$. pneumoniae, Salmonella spp, Citrobacter freundii, Enterobacter aerogenes, and Proteus mirabilis (George, 2009). Failure of empirical therapy is a frequent and common problem in infections caused by Amp-C producing isolates leading to significant morbidity and mortality (Harris PN., 2012, Conan MacDougall., 2011). There are no standard Clinical Laboratory Standards Institute (CLSI) guidelines to detect the presence of Amp-C $\beta$-lactamase. Amp-C is generally underreported which leads to therapeutic failures and spread of these resistant strains (George A 2009). Hence, there is an increased need to detect AmpC routinely in the laboratory.

\section{Objectives}

1) To detect AmpC $\beta$-lactamase enzyme production among Enterobacteriaceae isolated from various clinical samples

2) To compare different inducer substrate combinations for the detection of inducible Amp-C(iAmpC)

\section{Materials and Methods}

A total of 100 clinical isolates of Enterobacteriaceae from various samples like pus, urine, blood and sputum were included in the study. The isolates were identified by standard biochemical techniques.

\section{Detection of AmpC}

Constitutive AmpC (cAmpC) detected by inhibitor based method using Cefoxitin (CN) $(30 \mu \mathrm{g})$ and $\mathrm{CN}$ with Phenylboronic acid (PBA) (30/400 $\mu$ g) disks (Philip E. Coudron, 2005; Shoorashetty, 2011)

$120 \mathrm{mg}$ of phenylboronic acid was dissolved in $3 \mathrm{ml}$ of dimethyl sulfoxide. Three milliliters of sterile distilled water was added to this solution. Twenty microliters of the stock solution was dispensed onto disks containing $30 \mu \mathrm{g}$ of Cefoxitin. The boronic acid disk test was performed by inoculating Mueller-Hinton agar by the standard disk diffusion method and placing a disk containing $30 \mu \mathrm{g}$ of cefoxitin and a disk containing $30 \mu \mathrm{g}$ of Cefoxitin and $400 \mu \mathrm{g}$ of boronic acid onto the agar. Inoculated plates were incubated overnight at $35^{\circ} \mathrm{C}$. Bacteria that demonstrated a zone diameter around the disk containing cefoxitin and boronic acid that was $5 \mathrm{~mm}$ or greater than the zone diameter around the disk containing cefoxitin was considered an AmpC producer (Figure 1).

Double disk approximation method for the detection of iAmpC (Michael Dunne., 2005)

Disk diffusion susceptibility testing was performed as per standard guidelines. Inducers Imipenem (I) $(10 \mu \mathrm{g})$ and Cefoxitin (CN) $(30 \mu \mathrm{g})$, and substrates Cefotaxime (CTX) $(30 \mu \mathrm{g})$, Ceftazidime (CAZ) $(30 \mu \mathrm{g})$ and Piperacillin-Tazobactum $\quad(\mathrm{PT})(100 / 10 \mu \mathrm{g})$ were used. Various combinations tested were I/PT, I/CTX, I/CAZ, CN/CTX, CN/CAZ. 
Inducer/substrate disks were place on the surface of Mueller-Hinton agar plates at a distance of $25 \mathrm{~mm}$ apart. After incubation, zones of inhibition were measured on both the induced (adjacent to the inducer disk) and the uninduced side of the substrate disk from disk edge to zone edge. A test was considered positive if the zone of inhibition was reduced by $\geq 2 \mathrm{~mm}$ on the induced side of the substrate disk (Figure 2).

\section{Results and Discussion}

Out of 100 Enterobacteriaceae isolates, 52 were E.coli, 23 were Klebsiella spp, 19 were Enterobacter spp and 6 were Citrobacter spp. Amp C production was detected in $30 \%$ of isolates, $23 \%$ were constitutive and $7 \%$ were inducible (Figure 3). Commonest AmpC producer was Enterobacter sp with 7 (36.84\%) and 4(21.05\%), followed by E. coli $14(26.92 \%)$ and $3(5.76 \%)$ constitutive and iAmpC respectively. 2 (8.69\%) Klebsiella demonstrated only cAmpC (Figure 4). Sample wise distribution of $\mathrm{cAmpC}$ and $\mathrm{iAmpC}$ is depicted in Figure 5. I/PT combination detected all the 7 (100\%) iAmpC, others I/CTX and I/CAZ detected only $3(42.85 \%)$ isolates (Figure 6). Distribution of cAmpC and iAmpC among different strains in various clinical specimens is shown in Table 1.

Resistance to broad-spectrum beta lactams mediated by extended spectrum beta lactamases (ESBLs) and AmpC beta lactamases (AmpC $\beta \mathrm{Ls}$ ) enzymes is an increasing problem worldwide. In our study $23 \%$ of isolates were cAmpC and $7 \%$ of isolates were iAmpC. Shoorashetty and Nagarathnamma (2011) reported 33.5\% cAmpC and 7\% iAmpC. (Shoorashetty, 2011) Tan TY, Nq SY et al., (2009) reported 26\% cAmpC (Thean Yen Tan, 2009)

In our study, I/PT combination detected 100\% iAmpC. W. Michael Dunne, Jr. et al., in 2005, also found that I/PT combination provided the greatest sensitivity $(97.1 \%)$ to detect iAmpC (W. Michael Dunne., 2005). Bacteria expressing AmpC $\beta$ lactamases are of major clinical concern because these are usually resistant to all beta lactam antimicrobials, except for cefepime, cefpirome and carbapenems. The accurate detection of plasmid mediated AmpC is important to improve the clinical management of infection and to provide epidemiological data.

Table.1 Distribution of cAmpC and iAmpC among different strains in various clinical specimens

\begin{tabular}{|c|c|c|c|c|c|c|}
\hline \multirow[t]{2}{*}{ Samples } & \multicolumn{2}{|c|}{$\begin{array}{c}\text { E.coli, } \\
\mathbf{n}=52\end{array}$} & \multicolumn{2}{|c|}{$\begin{array}{c}\text { Klebsiella spp, } \\
\mathbf{n}=\mathbf{2 3}\end{array}$} & \multicolumn{2}{|c|}{$\begin{array}{c}\begin{array}{c}\text { Enterobacter spp } \\
\mathbf{n}=19\end{array} \\
\text {, }\end{array}$} \\
\hline & cAmpC & iAmpC & cAmpC & iAmpC & cAmpC & iAmpC \\
\hline Urine & $\begin{array}{c}9 \\
(17.3 \%)\end{array}$ & - & - & - & - & - \\
\hline Exudates & $\begin{array}{c}4 \\
(7.69 \%)\end{array}$ & $\begin{array}{c}1 \\
(1.92 \%)\end{array}$ & - & - & $5(26.31 \%)$ & $\begin{array}{c}2 \\
(10.52 \%)\end{array}$ \\
\hline Blood & - & $\begin{array}{c}2 \\
(3.84 \%)\end{array}$ & $\begin{array}{c}2 \\
(8.69 \%)\end{array}$ & - & $2(10.52 \%)$ & $\begin{array}{c}1 \\
(5.26 \%)\end{array}$ \\
\hline Sputum & $\begin{array}{c}1 \\
(1.92 \%)\end{array}$ & - & $\begin{array}{c}1 \\
(4.34 \%)\end{array}$ & - & - & $1(5.26 \%)$ \\
\hline Stool & - & - & - & - & - & - \\
\hline
\end{tabular}


Int.J.Curr.Microbiol.App.Sci (2019) 8(4): 848-854

Fig.1 Constitutive AmpC producer

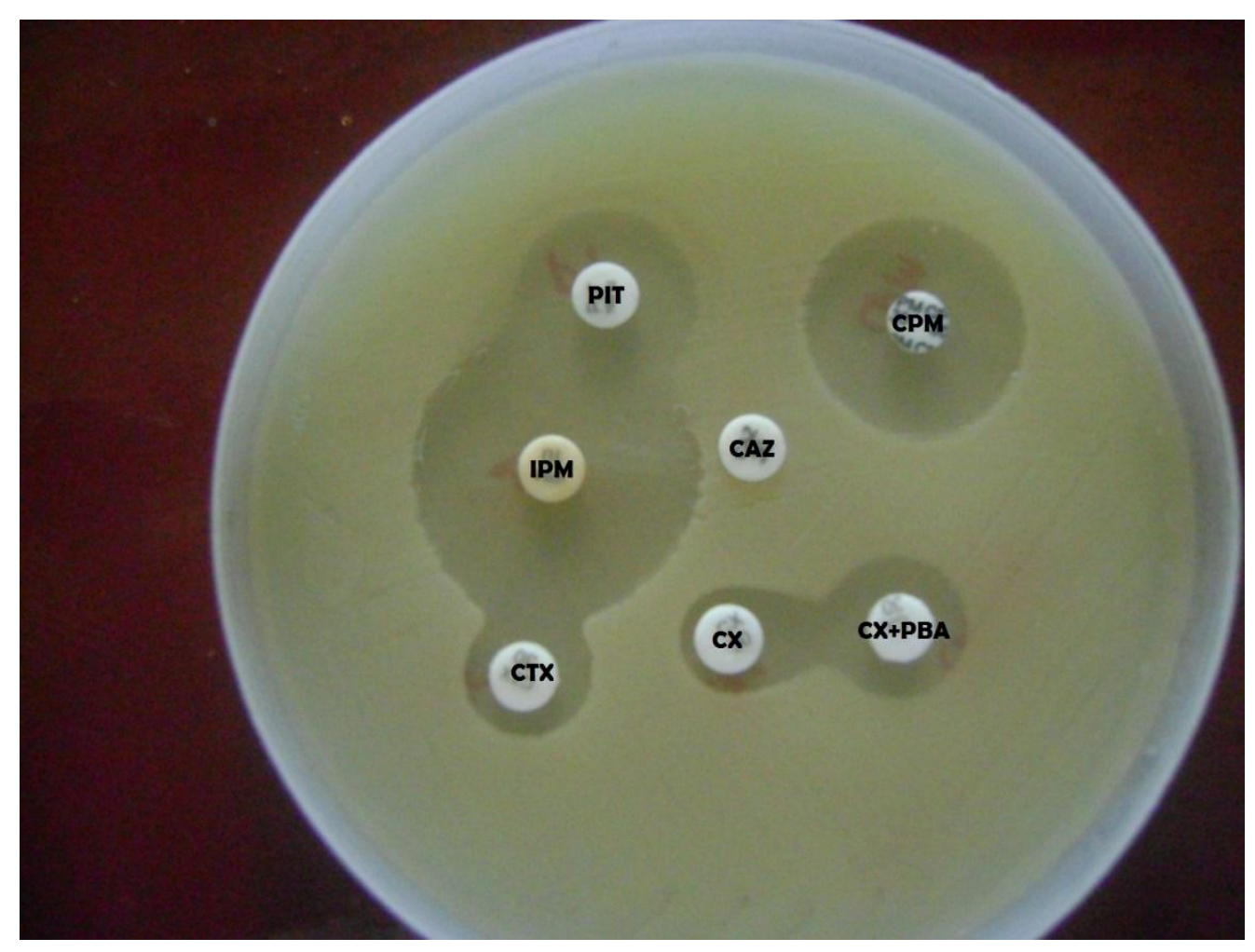

Fig.2 Inducible AmpC producer

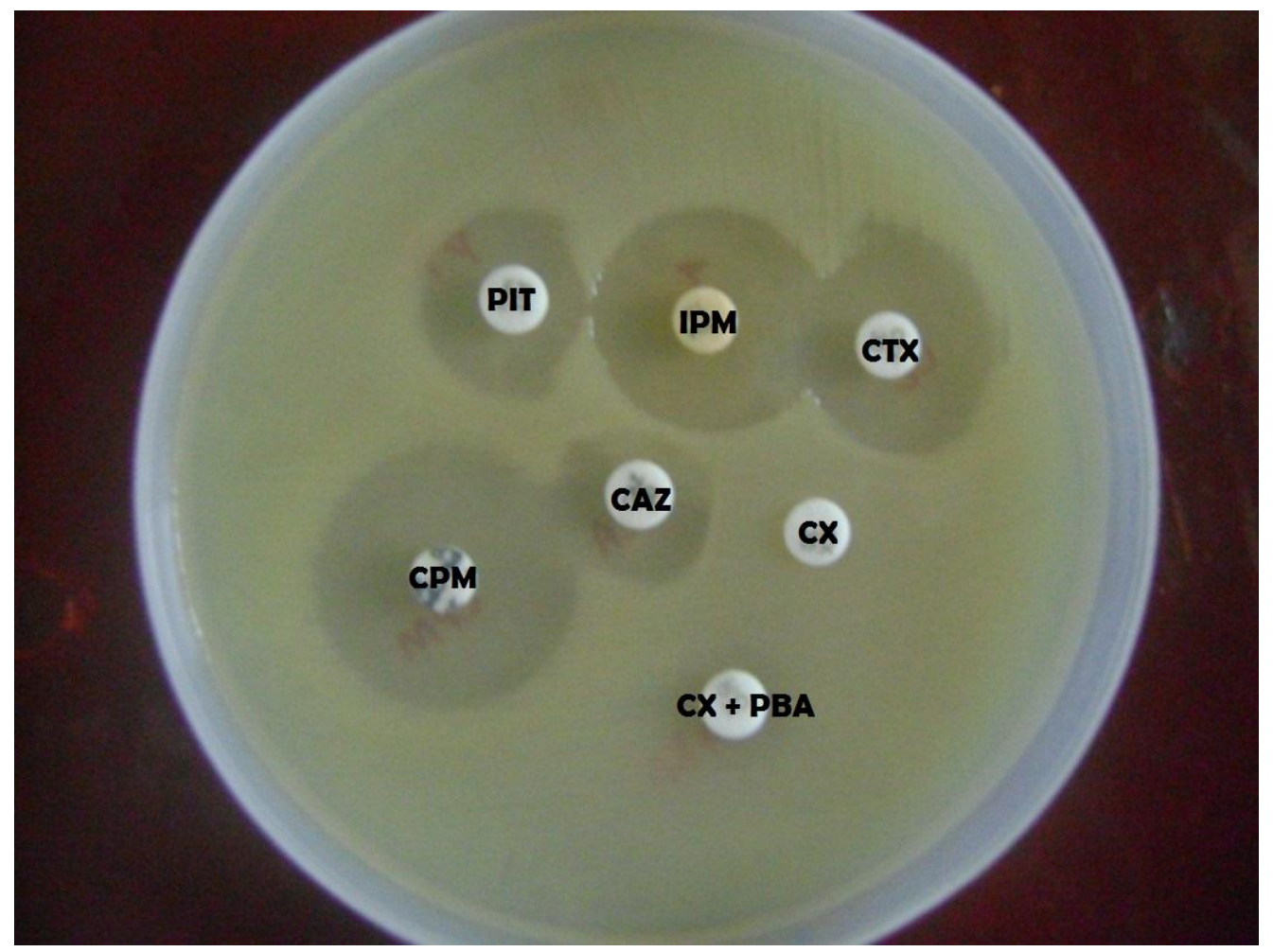


Fig.3 Percentage of AmpC producers

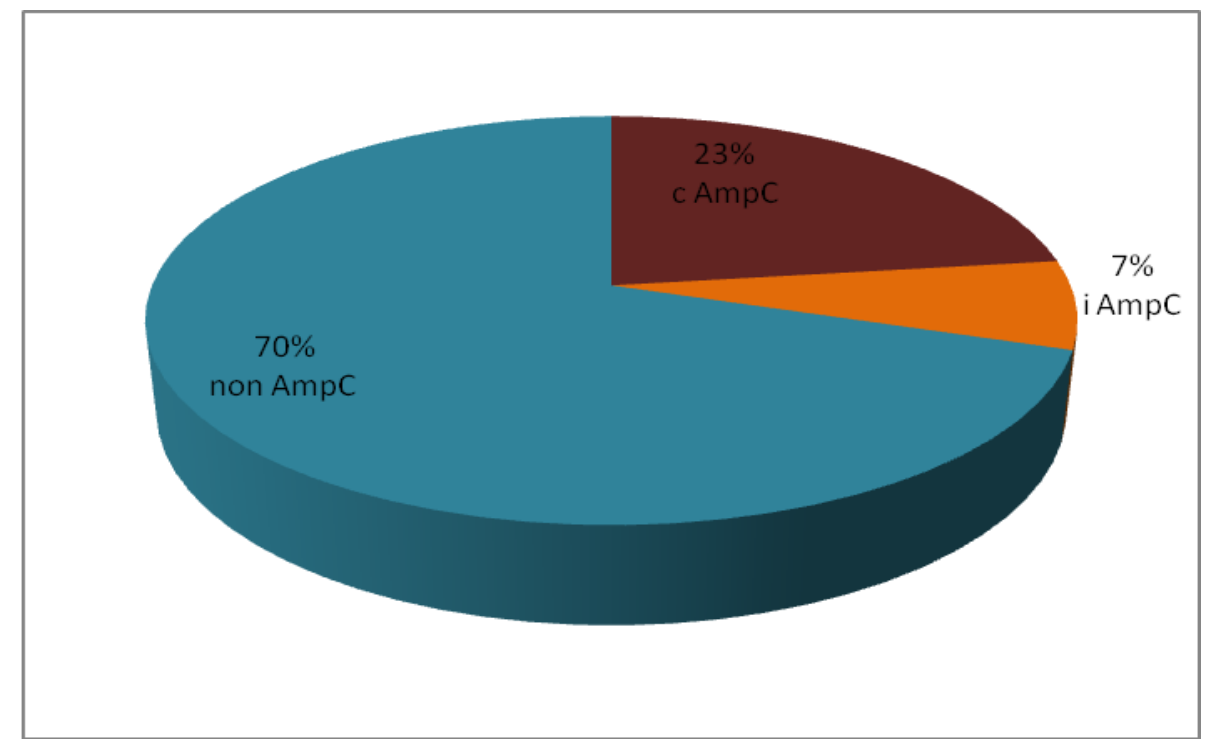

Fig.4 Distribution of cAmpC and iAmpC among various isolates

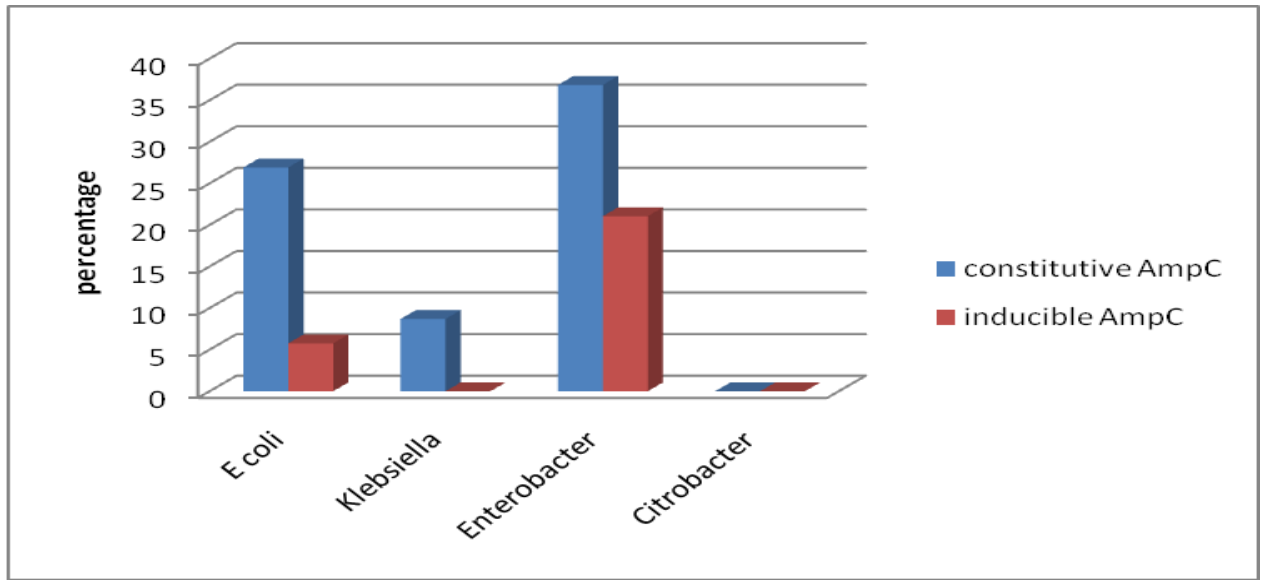

Fig.5 Sample wise distribution of AmpC isolates

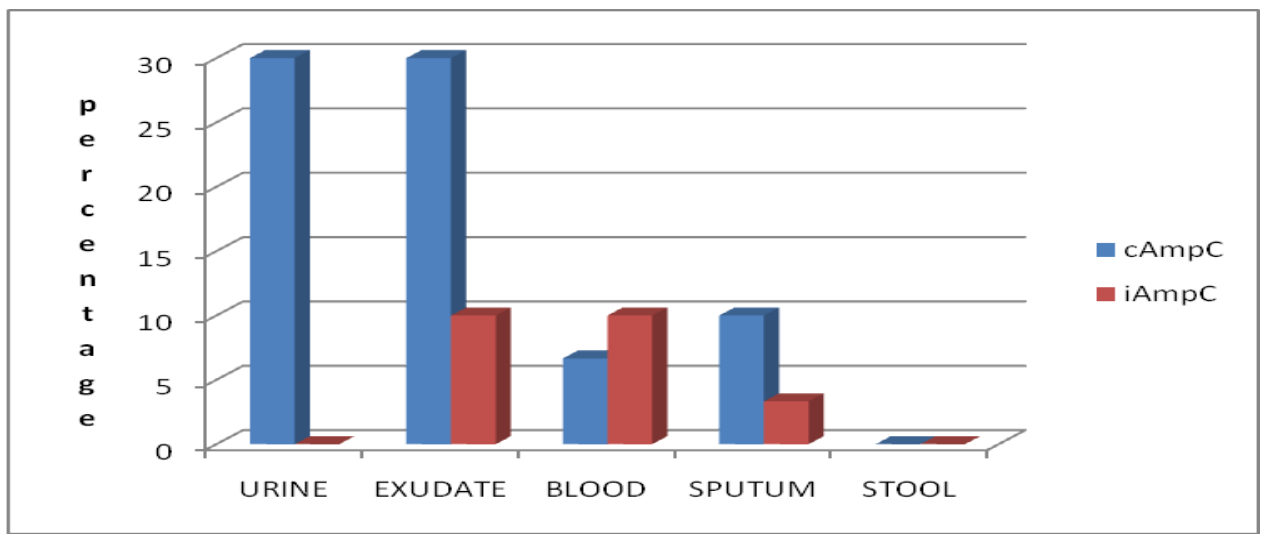


Fig.6 Comparison of various inducer substrate combinations for detection of iAmpC

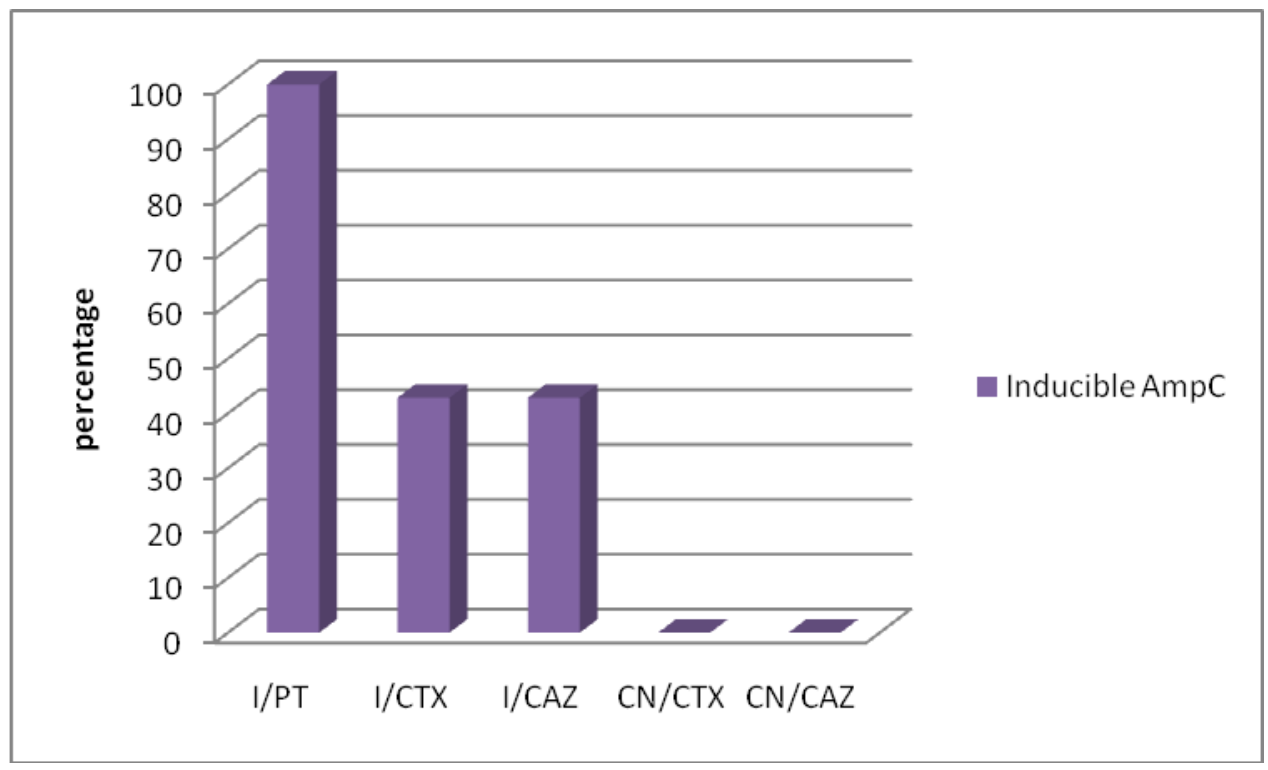

In conclusion, performing simple tests using boronic acid compounds as specific class $\mathrm{C} \mathrm{b}$ lactamase inhibitors for cAmpC and disk approximation test using I/PT combination for iAmpC enables clinical microbiology laboratories to report those strains producing class $\mathrm{C}$ b-lactamases thus helping physicians to select appropriate antimicrobial therapy. Determination of the prevalence of resistant strains is essential to formulate an effective antibiotic policy and hospital infection control measures.

\section{References}

AscelijnReuland, E., Teysir Halaby, John P. Hays. Plasmid-Mediated AmpC: Prevalence in Community-Acquired Isolates in Amsterdam, the Netherlands, and Risk Factors for Carriage. PLoS One. 2015; 10(1): e0113033

Conan MacDougall, PharmD, MAS. Beyond Susceptible and Resistant, Part I: Treatment of infections due to gramnegative organisms with inducible $\beta$ lactamases J Pediatr Pharmacol Ther. 2011 Jan-Mar; 16(1): 23-30.

George A. Jacoby Lahey Clinic, Burlington,
Massachusetts. AmpC -Lactamases. Clin Microbiol Rev, Jan. 2009, p. 161182.

Gunjan Gupta, Vibhor Tak and Purva Mathur. Detection of AmpC $\beta$ Lactamases in Gram-negative Bacteria J Lab Physicians. 2014 Jan-Jun; 6(1): 1-6.

Harris PN, Ferguson JK. Antibiotic therapy for inducible AmpC $\beta$-lactamaseproducing Gram-negative bacilli: what are the alternatives to carbapenems, quinolones and aminoglycosides? Int $\mathbf{J}$ Antimicrob Agents. 2012 Oct; 40(4):297-305.

Naveen Grover, Col, A.K. Sahni, Brig,and S. Bhattacharya. Therapeutic challenges of ESBLS and AmpC beta-lactamase producers in a tertiary care center. Med J Armed Forces India. 2013 Jan; 69(1): 4-10

Philip E. Coudron. Inhibitor-Based Methods for Detection of Plasmid-Mediated AmpC -Lactamases in Klebsiella spp., Escherichia coli, and Proteus mirabilis. J Clin Microbiol. 2005 Aug; 43(8): 4163-4167.

RM Shoorashetty, $\mathrm{T}$ Nagarathnamma, J Prathibha. Comparison of the boronic 
acid disk potentiation test and cefepimeclavulanic acid method for the detection of ESBL among AmpC-producing Enterobacteriaceae IJMM 2011; 29(3): 297-301

Thean Yen Tan, Lily Siew Yong Ng, Jie He, Tse Hsien Koh, and Li Yang Hsu. Evaluation of Screening Methods To Detect Plasmid-Mediated AmpC in Escherichia coli, Klebsiella pneumoniae, and Proteus mirabilis. Antimicrobial Agents and
Chemotherapy, Jan. 2009, p. 146-149 Vol. 53, No. 1.

W. Michael Dunne, Jr., Daniel J. Hardin. Use of Several Inducer and Substrate Antibiotic Combinations in a Disk Approximation Assay Format To Screen for AmpC Induction in Patient Isolates of Pseudomonas aeruginosa, Enterobacter spp., Citrobacter spp., and Serratia spp. J of Clin Microbiol, Dec. 2005, p. 59455949.

\section{How to cite this article:}

Sindhu Cugati and Lyra, P.R. 2019. Detection of Amp-C Beta Lactamase Enzyme Production among Enterobacteriaceae and Comparision of Different Inducer Substrate Combinations for Detection of Inducible Amp-C. Int.J.Curr.Microbiol.App.Sci. 8(04): 848-854.

doi: https://doi.org/10.20546/ijcmas.2019.804.095 